Proc. 13th International School on Theoretical Physics: Symmetry and Structural Properties of Condensed Matter

\title{
Analytical Skyrmion Solutions of the Non-Linear Sigma Model
}

\author{
S. TRIMPER* \\ Institute of Physics, Martin-Luther-University, Halle, Germany
}

\begin{abstract}
Motivated by studying quantum topological states we analyze the mesoscopic non-linear sigma model in two dimensions. We find systematically analytical solutions for the spatially depending magnetization density for arbitrary winding number under the constraint of topological protection. The methods allows also to get analytical expressions for other alignments such as a quadrupolar one. The inhomogeneous energy and skyrmion densities are calculated. Using a formulation of the local magnetization density in terms of a two component complex spinor field we get the related vector potential playing the role of the Berry curvature. The related magnetic field offers a spatial dependence which is completely different from electrodynamics. The dynamical stability of the solutions are discussed.
\end{abstract}

DOI: 10.12693/APhysPolA.135.1275

PACS/topics: magnetism, non-linear sigma model, topological states, skyrmions

\section{Introduction}

Skyrmions are stable field configurations which are protected by a topological constraint. Originally the concept had been introduced by Skyrme [1] in the context of particle physics following Heisenberg's idea to identify particles as inhomogeneous field configurations with finite and localized energy density. In a previous paper we have considered such a situation for the non-linear sigma model [2] based on [3]. A similar procedure has been applied by [4] and in more detail by $[5,6]$ for a review comparison [7]. The motivation of the present note is to offer a systematic way in getting analytical topologically protected skyrmion configurations for the isotropic sigma model without any additional chiral terms. Using a representation of the magnetization density in terms of a complex spinor field [8] the system reveals a gauge potential which adopts the Berry connection well established in quantum systems. The related magnetic field playing the role of the Berry curvature can be calculated for certain skyrmion alignments such as a configuration with arbitrary winding number and for instance a quadrupolar arrangement. Moreover, we discuss the dynamical stability of the analytical solutions.

\section{Sigma model and gauge potential}

The nonlinear-sigma model is a classical continuous version of the quantum Heisenberg model. It is formulated in terms of a local real three component magnetization field $\boldsymbol{n}(\boldsymbol{x}, t)$ with a fixed length which is related to the conservation of the total spin [9]. The Hamiltonian reads in two-dimensional case

$$
H=\int \mathrm{d}^{2} x h(\boldsymbol{x}), \quad h(\boldsymbol{x})=\frac{J}{2}(\nabla \boldsymbol{n})^{2} .
$$

\footnotetext{
*e-mail: steffen.trimper@physik.uni-halle.de
}

Notice that chirality in form of a term $\boldsymbol{n} \cdot \nabla \times \boldsymbol{n}$ [10-12] is not included in our model. Because of $\boldsymbol{n}(\boldsymbol{x},-t)=-\boldsymbol{n}(\boldsymbol{x}, t)$ the energy density $h(\boldsymbol{x}, t)$ is invariant under time reflection. The constraint $\boldsymbol{n}^{2}=1$ implies a local sphere in spin space, the total surface of which is a topologically protected invariant

$$
Q=\frac{1}{4 \pi} \int \mathrm{d}^{2} x \boldsymbol{n} \cdot\left(\partial_{x} \boldsymbol{n} \times \partial_{y} \boldsymbol{n}\right)=\frac{1}{4 \pi} \int \mathrm{d}^{2} x q(\boldsymbol{x}) .
$$

Here we have introduced the density of the topological invariant (skyrmion density) $q(\boldsymbol{x})=q(x, y)$ on the $x-y$ plane. The topological invariant $Q$ is changed under time reflection $Q(-t)=-Q(t)$ whereas the energy is invariant under time reflection. Moreover, $Q$ changes its sign in case the coordinates $x$ and $y$ are interchanged whereas the energy density remains invariant. This fact leads to a degeneration of skyrmion solutions discussed later. The Hamiltonian is altered under local rotation $\boldsymbol{n}(\boldsymbol{x}) \rightarrow \boldsymbol{n}(\boldsymbol{x})+\boldsymbol{\Theta}(\boldsymbol{x}) \times \boldsymbol{n}(\boldsymbol{x})$ to

$$
\delta H=J \int \mathrm{d}^{d} x \Pi_{\mu}^{\alpha} \partial_{\mu} \Theta_{\alpha}, \text { with } \Pi_{\nu}^{\alpha}=\varepsilon_{\alpha \beta \gamma} n_{\beta} \partial_{\nu} n_{\gamma} .
$$

The tensor $\Pi_{\mu}^{\alpha}, \alpha=1, \ldots, n=3, \mu=1, \ldots, d=2$ is the spin-current density which appears due to the local distortion. In terms of $\boldsymbol{\Pi}_{\mu}=J\left(\boldsymbol{n} \times \partial_{\mu} \boldsymbol{n}\right)$ the topological density in Eq. (2) can be expressed by

$$
q(\boldsymbol{x})=\frac{1}{2}\left(\partial_{\mu} n_{\alpha}\right)\left(\varepsilon_{\nu \mu} \Pi_{\nu}^{\alpha}\right) \equiv \frac{1}{2}\left(\partial_{\mu} n_{\alpha}\right) \Gamma_{\mu}^{\alpha} .
$$

Here the quantity $\Gamma_{\mu}^{\alpha}$ is related to the spin current and fulfills

$$
\left(\partial_{\mu} n_{\alpha}\right)^{2}=\left(\Gamma_{\mu}^{\alpha}\right)^{2}
$$

To reflect the properties of the underlying spins as complex quantities and to separate the fields and the local gauge potential let us introduce $[8,10]$ :

$$
n_{\alpha}=\boldsymbol{\psi}^{\dagger} \sigma_{\alpha} \boldsymbol{\psi}, \quad \boldsymbol{\psi}^{\dagger}=\left(\psi_{1}^{*}, \psi_{2}^{*}\right) .
$$

Here $\sigma_{\alpha}$ are the (complex) Pauli-matrices whereas $\boldsymbol{\psi}(\boldsymbol{x})$ is a two-component complex spinor field with the properties

$$
|\boldsymbol{\psi}|^{2}=1, \quad \rightarrow \quad \boldsymbol{\psi} \partial_{\mu} \boldsymbol{\psi}^{\dagger}=-\boldsymbol{\psi}^{\dagger} \partial_{\mu} \boldsymbol{\psi}
$$


After a straightforward calculation the Hamiltonian in Eq. (1) reads

$$
H=2 J \int\left(\left|\partial_{\mu} \psi\right|^{2}-A_{\mu}^{2}\right) \mathrm{d}^{2} x,
$$

where the vector gauge potential $\boldsymbol{A}$ is given by

$$
A_{\mu}=-\frac{\mathrm{i}}{2}\left[\psi_{i}^{*} \partial_{\mu} \psi_{i}-\psi_{i} \partial_{\mu} \psi_{i}^{*}\right] \equiv-\mathrm{i} \boldsymbol{\psi}^{\dagger} \partial_{\mu} \boldsymbol{\psi}
$$

In the quantum context the potential $\boldsymbol{A}$ is called the Berry connection, for a recent review see [13]. The related magnetic field for $d=2$ is given by

$$
B_{z}=\epsilon_{\mu \nu} \partial_{\mu} A_{\nu}=\partial_{x} A_{y}-\partial_{y} A_{x} \text {. }
$$

This field $B_{z}$ corresponds to the Berry curvature. Under the local gauge transformation of the complex field $\boldsymbol{\psi}$ with a real function $\Lambda(\boldsymbol{x})$ according to

$$
\psi_{i} \rightarrow \psi_{i}^{\prime}=\mathrm{e}^{\mathrm{i} \Lambda(\boldsymbol{x})} \psi_{i}
$$

the vector potential $\boldsymbol{A}_{\mu}$ is transformed to

$$
A_{\mu}^{\prime}=A_{\mu}(\boldsymbol{\psi})+\partial_{\mu} \Lambda \text {. }
$$

Introducing the covariant derivative $D_{\mu}=\partial_{\mu}-i A_{\mu}$ one can express $H$ and the topological invariant as

$$
\begin{aligned}
& H=2 J \int \mathrm{d}^{d} x\left(D_{\mu} \psi_{i}\right)^{*}\left(D_{\mu} \psi_{i}\right), \\
& Q=\frac{\mathrm{i}}{2 \pi} \int \mathrm{d}^{2} x \epsilon_{\mu \nu} D_{\mu} A_{\nu}
\end{aligned}
$$

Due to the gauge transformation the number of degrees of freedom remains unchanged.

\section{Skyrmion solutions in $d=2$}

A local distortion of the magnetization field $\boldsymbol{n}(\boldsymbol{x})$ is related to both, a change of the energy density $h(\boldsymbol{x})$ and simultaneously to a change of the topological density $q(\boldsymbol{x})$. To reveal a relation between $h(\boldsymbol{x})$ and $q(\boldsymbol{x})$ let us consider the inequality

$$
R(\boldsymbol{x})=\left(\partial_{\mu} n_{\alpha} \mp \Gamma_{\mu}^{\alpha}\right)^{2} \geq 0 .
$$

Using Eqs. (4), (5) the local condition follows

$$
h(\boldsymbol{x}) \mp J q(\boldsymbol{x}) \geq 0 \text {. }
$$

This relation is a local one indicating the above mentioned strong correlation between the local energy density and the local skyrmion density. In case of $d=2$, i.e. the magnetization field is in a plane, such as $x-y$ plane, we integrate the last relation finding the global relationship $H \geq \pm 4 \pi J Q$. From here we conclude the global condition for the minimal energy of the topologically protected states

$$
H_{\min }=4 \pi J|Q| \text {. }
$$

The last relation is realized for $R(\boldsymbol{x})=0$ in Eq. (14):

$$
\partial_{\mu} n_{\alpha}= \pm \Gamma_{\mu}^{\alpha} \text {. }
$$

Using the representation

$$
\psi_{1}=\mathrm{e}^{\mathrm{i} \theta_{1}} \cos \varphi, \quad \psi_{2}=\mathrm{e}^{\mathrm{i} \theta_{2}} \sin \varphi .
$$

Equation (17) is consistent with

$$
2 \partial_{x} \varphi=\mp \sin 2 \varphi \partial_{y} \theta, \quad 2 \partial_{y} \varphi= \pm \sin 2 \varphi \partial_{x} \theta .
$$

From here we conclude that the phase $\theta=\theta_{1}-\theta_{2}$ in the realization in Eq. (18) fulfills $\nabla^{2} \theta=0$ with the solution

$$
\theta(x, y)=\sum_{q} q \tan ^{-1} \frac{y-y_{q}}{x-x_{q}}, \quad q= \pm 1, \pm 2 \ldots
$$

The integer factor $q$, the winding number, appears because $\theta$ is an angle. Inserting this solution in Eq. (19) one finds the solution for the azimuthal angle $\varphi$ as

$$
\varphi(x, y)=\tan ^{-1} \prod_{q}\left(\frac{r_{q}}{a}\right)^{\mp q} .
$$

The solution given in Eqs. (20), (21) had been already obtained in [2]. Here $a$ is a lower cut-off, $r_{q}^{2}=\left(x-x_{q}\right)^{2}+$ $\left(y-y_{q}\right)^{2}$ with the constants $x_{q}, y_{q}$ which will be identified with position of the skyrmions. The solution reflects the already mentioned topological degeneracy, that the interchange $y \leftrightarrow x$ is related to altering $q \leftrightarrow-q$ After gauge transformation Eq. (12) the Berry connection is

$$
A_{\mu}=\left(\partial_{\mu} \theta\right) \cos ^{2} \varphi \text {. }
$$

\section{Solution with arbitrary winding number $q$}

Let us illustrate the approach for an arbitrary $q$ which leads due to Eqs. (20) and (21) to

$$
\theta(x, y)=q \tan ^{-1} \frac{y}{x}, \quad \varphi(x, y)=\tan ^{-1}\left(\frac{r}{a}\right)^{\mp q} .
$$

Here $r^{2}=x^{2}+y^{2}$ is the distance from the position of the skyrmion, the core of which is situated at the origin $x_{q}=y_{q}=0$. In terms of polar coordinates $x=r \cos \alpha, y=r \sin \alpha$ in the coordinate space $d=2$ the order parameter field is

$$
\begin{aligned}
& n_{x}(r, \alpha)=\frac{2(r a)^{q}}{r^{2 q}+a^{2 q}} \cos (q \alpha), \\
& n_{y}(r, \alpha)=-\frac{2(r a)^{q}}{r^{2 q}+a^{2 q}} \sin (q \alpha), \\
& n_{z}(r, \alpha)= \pm \frac{r^{2 q}-a^{2 q}}{r^{2 q}+a^{2 q}} \rightarrow \pm 1 \text { for } r \rightarrow \infty .
\end{aligned}
$$

As an example the magnetization field is depicted in Fig. 1 for $q=1$.

At the position of the origin of the skyrmion at $x=y=$ 0 the magnetization field is $\boldsymbol{n}(0,0)=(0,0,-1)$ for the upper sign in Eq. (17) and $\boldsymbol{n}(0,0)=(0,0,+1)$. As indicated in Eq. (24) the spin flips to $\boldsymbol{n}(r \rightarrow \infty)=(0,0, \pm 1)$. The topological density according to Eq. (2) reads

$$
q(r)= \pm \frac{q^{2} a^{2 q} r^{2 q-2}}{\pi\left(r^{2 q}+a^{2 q}\right)^{2}} .
$$

The integration leads to $Q=\int q(r) \mathrm{d} r r \mathrm{~d} \alpha= \pm q$. The skyrmion density $q(\boldsymbol{x})$ and simultaneously the energy density offers a pronounced peak around the origin which is shown in Fig. 2.

In the same manner we find the gauge potential $\boldsymbol{A}$ which gives rise to the Berry curvature.

$$
B_{z}=\partial_{x} A_{y}-\partial_{y} A_{x}= \pm \frac{2 q^{2}(a r)^{2 q}}{r^{2}\left(r^{2 q}+a^{2 q}\right)^{2}} .
$$

The field $B_{z} \simeq q^{2} r^{-2(q+1)}$ is totally different from conventional electrodynamics. The related current $\operatorname{rot} \boldsymbol{B}=\tilde{\boldsymbol{j}}$ offers in polar coordinates a nonzero component in angle direction 


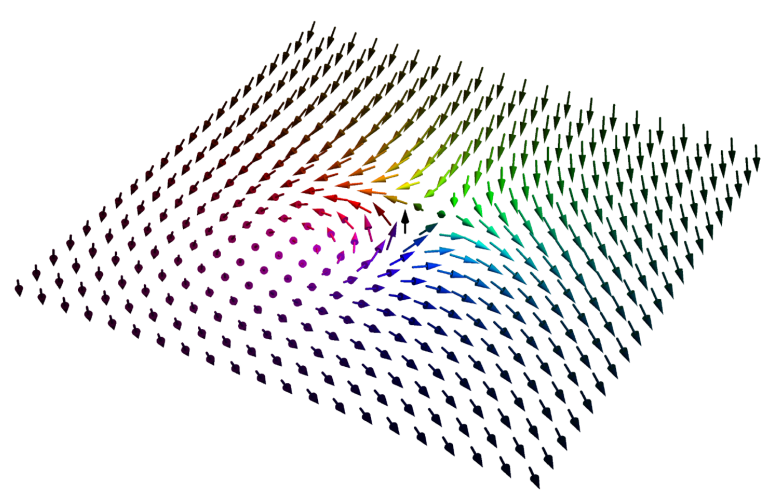

Fig. 1. A single skyrmion with $q=1$.

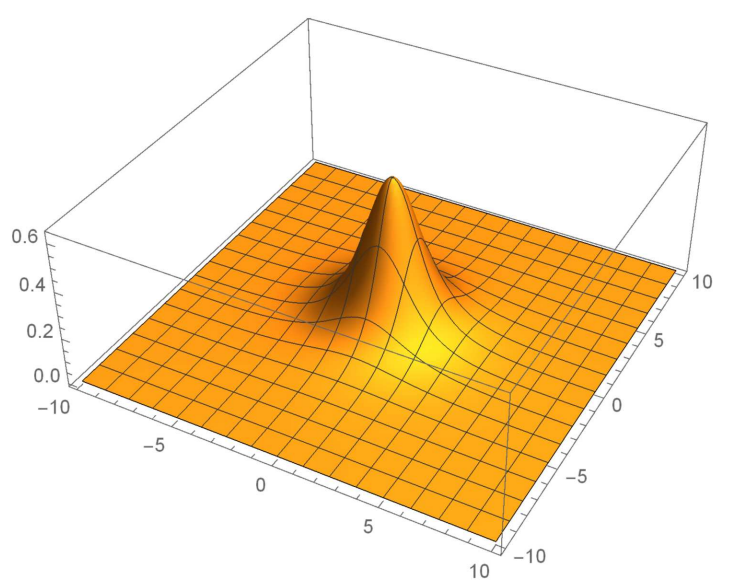

Fig. 2. Skyrmion density $q(x, y)$ on the $x-y$ plane with winding number $q=1$.

$$
\tilde{j}_{\alpha}= \pm \frac{4 q^{2}(a r)^{2 q}}{r^{3}\left[r^{2 q}+a^{2 q}\right]^{3}}\left((1+q) r^{2 q}+(1-q) a^{2 q}\right) .
$$

This current is directly related to the topological current introduced in Eq. (32). We have discussed a lot of different analytical solutions such as dipolar configuration with $q= \pm 1$ or with both $q=1$, different linear alignments, hexagonal configurations and other ones. Here we present the quadrupolar order with 4 alternating winding numbers. Two skyrmions with $q=1$ and distance $2 a$ are along the $x$-axis whereas two skyrmions with $q=-1$ along the $y$-axis and likewise distance $2 a$. The analytical solution is

$$
\begin{aligned}
& n_{x}=\frac{r^{4}-a^{4}}{r^{4}+a^{4}}, \quad n_{y}=-\frac{4 a^{2} x y}{r^{4}+a^{4}}, \\
& n_{z}= \pm \frac{2 a^{2}\left(y^{2}-x^{2}\right)}{r^{4}+a^{4}}
\end{aligned}
$$

The solution is shown in Fig. 3 .

The $B$ field is isotropic and simply given by $B_{z}=$ $\pm 8 a^{4} r^{2}\left(r^{4}+a^{4}\right)^{-2}$. The density $q(\boldsymbol{x})$ is given by

$$
q(\boldsymbol{x})=\frac{16 a^{4} r^{2}}{\left(r^{4}+a^{4}\right)^{2}}, \quad Q=2 .
$$

and is shown in Fig. 4.

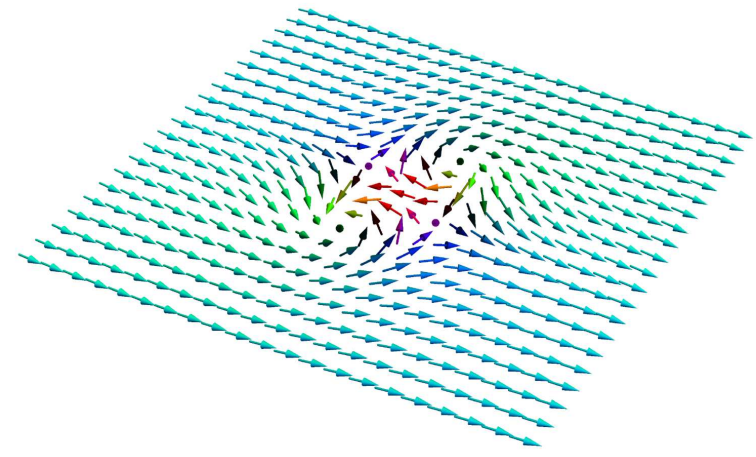

Fig. 3. Quadrupolar configuration with alternating winding numbers $q= \pm 1$.

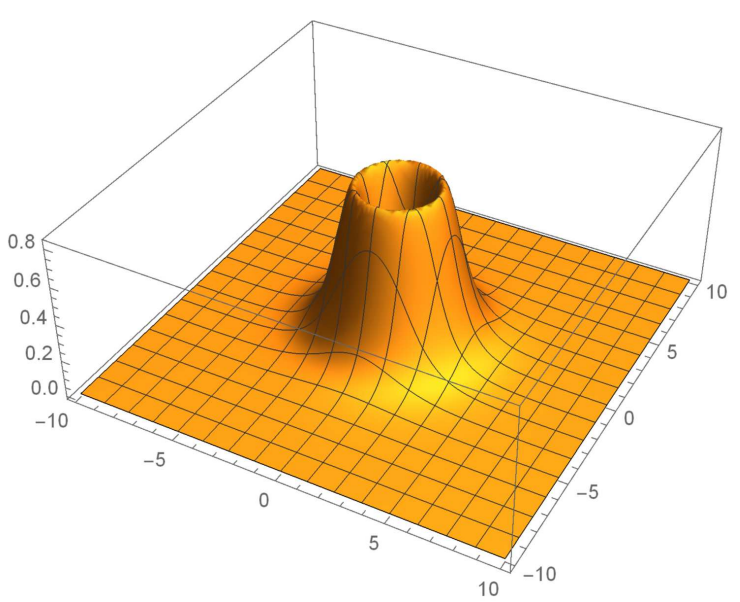

Fig. 4. Skyrmion density $q(x, y)$ on the $x-y$ plane for a quadrupolar configuration.

\section{Dynamics}

In this section we discuss the dynamical stability of the skyrmion solution. In case the magnetization field fulfills the Landau-Lifshitz-Gilbert equation

$$
\partial_{t} \boldsymbol{n}+J \partial_{\nu} \boldsymbol{\Pi}_{\nu}=-\boldsymbol{\Omega}^{(n)}, \text { with } \boldsymbol{\Omega}^{(\mathrm{n})}=+\gamma, \dot{\mathrm{n}} \times \mathrm{n} .
$$

The continuity equation for the density $u(\boldsymbol{x}, t)=h(\boldsymbol{x}, t)$ or $q(\boldsymbol{x}, t)$ reads

$$
\frac{\partial u(\boldsymbol{x}, t)}{\partial t}+\operatorname{divj}^{(\mathrm{u})}=-\Omega^{(\mathrm{u})}
$$

with $\Omega^{(e)}=J \alpha \boldsymbol{n} \cdot(\nabla \boldsymbol{n} \times \nabla \dot{\boldsymbol{n}}), \quad \Omega^{(q)}=J \alpha(\nabla \dot{\boldsymbol{n}} \times \nabla \boldsymbol{n})_{z}$ The related currents are

$$
j_{\mu}^{(q)}=J \epsilon_{\alpha \beta} \partial_{\alpha} n_{\gamma} \partial_{\mu \beta} n_{\gamma} \cdot j_{\mu}^{(h)}=J \epsilon_{\alpha \beta \gamma} \partial_{\mu} n^{\alpha} n_{\beta} \partial_{\nu \nu} n_{\gamma} .
$$

For arbitrary $q$ the topological current is in polar coordinates

$$
\boldsymbol{j}^{(q)}(\boldsymbol{x})=2 J \frac{4(r a)^{2 q} q^{2}\left[(q+1) r^{2 q}+(1-q) a^{2 q}\right]}{r^{3}\left(r^{2 q}+a^{2 q}\right)^{3}} \boldsymbol{e}_{\alpha}
$$

which is up to a factor the above introduced fictitious current $\tilde{\boldsymbol{j}}$ (see Eq. (27)). Insofar the topological current is directly related to the Berry field according to Eq. (27). 
The topological current is free of sources, i.e. $\nabla \cdot \boldsymbol{j}^{(q)}=0$. Due to the continuity equation Eq. (31) for zero damping this fact reflects the dynamical stability of the skyrmion solutions. In the same manner we get for the solution with arbitrary $q$ the relation $\partial_{\mu} \Pi_{\mu}^{\alpha}=0$ which is accordance with Eq. (30) without zero damping. Again the dynamical stability of skyrmion configurations is guaranteed. The inclusion of damping effects will be discussed elsewhere.

\section{Conclusion}

We have analyzed the mesoscopic non-linear sigma model in order to find topologically protected inhomogeneous solutions for the magnetization field, the energy, and the skyrmion density. The method can be extended to get more refined skyrmion configurations. Moreover, we have discussed the dynamical behavior of the solution. The solutions presented are dynamically stable.

\section{Acknowledgments}

I gratefully acknowledge discussions with Ingrid Mertig, Alexander Mock, and Börge Göbel.

\section{References}

[1] T.H.R. Skyrme, Nucl. Phys. 31, 556 (1962).

[2] S. Trimper, Phys. Lett. A 70, 114 (1979).

[3] A.A. Belavin, A.M. Polyakov, JETP Lett. 22, 245 (1975).

[4] A.N. Bogdanov, D.A. Yablonskii, JETP 68, 101 (1989).

[5] S. Mülbauer, B.B.F. Jonietz, C. Pfleiderer, A. Rosch, A. Neubauer, R. Georgii, P. Bni, Science 323, 915 (2009).

[6] N. Nagaosa, Y. Tokura, Nat. Nanotechnol. 8, 899 (2013).

[7] G. Tatara, Physica E 106, 208 (2019).

[8] N. Nagaosa, Quantum Field Theory in Strongly Correlated Electronic Systems, Springer, New York 1999.

[9] A.M. Polyakov, Phys. Lett. B 59, 79 (1975).

[10] J.H. Han, J. Zang, Z. Yang, J.H. Park, N. Nagaosa, Phys. Rev. B 82, 094429 (2010).

[11] W.T. Hou, J.X. Yu, M. Daly, J. Zang, Phys. Rev. B 96, 140403 (2017).

[12] H. Shimada, K. Takahashi, H.T. Ueda, Phys. Rev. B 97, 224424 (2018).

[13] S. Rachel, Rep. Prog. Phys. 81, 116501 (2018). 